Revista Electrónica de Investigación en Ciencias Económicas

Abriendo Camino al Conocimiento

Facultad de Ciencias Económicas, UNAN-Managua

\title{
EL SALARIO MÍNIMO Y SU RELACIÓN CON LA CANASTA BÁSICA
}

Fátima Ninoska Guzmán Cisneros ${ }^{1}$ Estudante 2do año Carrera de Economia

UNAN-Managua guzfatima@gmail.com

Cristhiam Elieth Robleto Acevedo Estudante 2do año Carrera de Economia UNAN-Managua Crist robleto@hotmail.com

María Félix Andino Moran Estudante 2do año Carrera de Economia UNAN-Managua maria.fam.com@hotmail.es

Fecha recepción: octubre 23 del 2014 Fecha aceptación: noviembre 11 del 2014

Palabras claves: Salario Mínimo, Canasta Básica, Índices de precios al consumidor Inflación, Crecimiento Económico

Keywords: Minimum Wage, Basketball Basic, Consumer Price Index, Inflation Economic Growth.

ISSN: $2308-782 X$

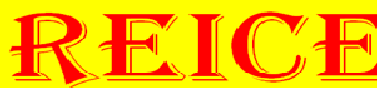

Revista Electrónica de Investigación en Ciencias Económicas http://revistacienciaseconomicas.unan.edu.ni revistacienclaseconomicas@gmall.com revistarucfa@unan.edu.ni

Resumen

La finalidad de este ensayo es dar una explicación sobre la relación de las variables Canasta Básica y Salario Mínimo. Nuestro propósito es recalcar en

\footnotetext{
${ }^{1}$ Ensayo presentado en la X JUDC Facultativo, FFCCEE, UNAN-Managua, realizado del 22 al 23 de octubre 2014
}

REICE Vol. 2, No. 4, julio-diciembre 2014 la brecha que existe entre ambas variables. La metodología de este artículo es descriptivo analítico en donde se evaluara el comportamiento de las variables determinantes del salario mínimo. Desde el punto de vista económico en Nicaragua la problemática es la siguiente: bajos salarios con altos niveles de precios de los productos de la canasta básica. Con este resultado es evidente, que se producirá un incremento mayor en la brecha de salario mínimo y de costo de la canasta básica, haciendo cada día una utopía un salario mínimo igual al costo de la canasta básica.

\section{Abstract}

The purpose of this essay is to explain the relationship of the basic basket variables and minimum wage. Our purpose is to emphasize the gap between the two variables. The methodology of this paper is descriptive analytic where the behavior of the variables determining the minimum wage be evaluated. From the economic point of view in Nicaragua the problem is this: low wages with high prices of basic food products. With this result it is evident that further developments will occur in the gap of minimum wage and cost of the basket, making every day a utopia minimum wage equal to the cost of the basket. 
Introducción

Facultad de Ciencias Económicas, UNAN-Managua

Una familia promedio necesita, para cubrir sus necesidades básicas a partir de sus ingresos, un conjunto de bienes y servicios considerados en una canasta básica. En Nicaragua la Canasta Básica tiene todos los productos necesarios para poder tener una vida sana, tanto física como mentalmente. Sin embargo, es un tema de mucha controversia en nuestro país, ya que el salario que por lo general un trabajador obtiene producto de su fuerza de trabajo es muy bajo para el alto precio de la canasta básica. Utilizaremos el salario mínimo ya que aproximadamente el $60 \%$ de la PEA gana el nivel de salario mínimo establecido oficialmente por el Estado.

El salario mínimo es un ejemplo de precio mínimo utilizado en las ecuaciones de oferta y demanda de trabajo, esta consiste en que la cantidad ofrecida de trabajo es superada por la demanda y en consecuencia existe como resultado el desempleo. (Véase anexo\#1).

El propósito de este trabajo es analizar la relación entre el salario mínimo y la canasta básica para poner en práctica nuestros conocimientos en metodología de la investigación, así como también, mostrar una nueva perspectiva de análisis entre las variables de inflación y canasta básica y estudiar la evolución de la inflación y el crecimiento económico en el periodo 2012-2013.

Este tema es de gran importancia debido a los comportamientos que han tenido las variables salario mínimo y canasta básica en los últimos años, de modo que han causado tanto efectos positivos como negativos y es necesaria una valoración para la mejora del comportamiento de estas variables.

La metodología de este artículo es descriptivo analítico en donde se evaluara el comportamiento de las variables determinantes del salario mínimo (inflación y crecimiento económico), adicionalmente se evaluará la estimación del IPC y su índice Laspayres y el PIB real y su índice de paasche. Para ello se utilizó datos del BCN, MITRAB e INIDE. 
Revista Electrónica de Investigación en Ciencias Económicas

Abriendo Camino al Conocimiento

Facultad de Ciencias Económicas, UNAN-Managua

Los resultados obtenidos muestra que en Nicaragua el problema es el uso de los índices de precio Laspeyres (utilizado en IPC y este a su vez para la inflación) y índice Paasche (utilizado para estimar el deflactor del PIB y este a su vez para determinar el crecimiento económico).Estos índices están preparados para determinar parámetros bajos, tanto en la inflación como en el crecimiento económico, lo que restringe las negociaciones semestrales de salario mínimo entre los empresarios , Gobierno y trabajadores. Con este resultado es evidente, que se producirá un incremento mayor en la brecha de salario mínimo y de costo de la canasta básica, haciendo cada día una utopía un salario mínimo igual al costo de la canasta básica.

Material y métodos

La metodología utilizada ha consistido en un análisis descriptivo, explicativo y correlacional de la evolución del desabastecimiento de frijol rojo en Nicaragua, a partir de la información proporcionada por el Banco Central de Nicaragua (BCN), Ministerio de Agricultura y Ganadería (MAG), Ministerio de Fomento, Industria y Comercio (MIFIC).

\section{Resultado y Discusión}

Ahora como se determina el salario, se necesita que el obrero regrese a la fábrica, oficina o lugar de trabajo cada día y por ello se determina el salario por el valor de los medios de subsistencia del obrero y de su familia (Canasta básica alimentaria o canasta básica vital), por el precio de los bienes y servicios que el obrero y su familia deben consumir para sobrevivir. ${ }^{1}$

Por lo antes dicho, podemos ver que el salario mínimo es uno de los temas más polémicos y controversiales en la práctica económica y social de cualquier parte de Nicaragua. Uno de los elementos que más se relaciona al analizar el salario mínimo es la Canasta básica, la cual está formada por 3 componentes que son alimentación, gastos del hogar y vestuario. 
Revista Electrónica de Investigación en Ciencias Económicas

Abriendo Camino al Conocimiento

Facultad de Ciencias Económicas, UNAN-Managua

La canasta básica de Nicaragua está conformada por 53 productos alimenticios y no alimenticios actualizada con precios de IPC.

Para el año 2012 según estadística del INIDE el rubro alimentación conforma un 63\% que en comparación al siguiente año (año 2013) es igual a un 63\% lo cual, significa que el incremento fue solamente de 582.57 córdobas, de modo que no se ve diferenciado el porcentaje de ambos años. Los bienes de mayor proporción fueron la posta de res, el arroz, la leche, el queso seco y la tortilla.

Para el componente gastos del hogar del año 2012 el costo fue de $26 \%$ y en comparación año 2013 fue de 25\% obteniendo una diferencia de 1\% más que el año anterior, lo que significa que los precios de los gastos del hogar incrementaron 50.28 córdobas. Los componentes de mayor precio fueron: el alquiler, el gas, la luz y el jabón de lavar ropa.

En el 2012 el rubro vestuario represento un 11\% y en el año 2013 fue de $12 \%$ lo que significa que en este componente se incrementó en $1 \%$ que en córdobas se estima un equivalente a 177.23 córdobas. Así logramos observar que a medida que el tiempo pasa el costo de la canasta básica aumenta también.

Dentro del componente vestuario las mujeres y niñas mayores de 10 años representan la porción más alta del rubro (un $40 \%$ ), en relación al gasto en el vestuario de los varones y niños ya que las familias gastan un poco más en accesorios para niñas que de niños.

El salario mínimo promedio de todos los sectores económicos en Nicaragua, es de C \$ 4,256.23 córdobas (463 córdobas más que en 2012). El sector agropecuario posee el menor salario mínimo con C\$2566.9 córdobas para 2013 (23\% del costo de la canasta básica), le siguen en el sector Gobierno Central con $\mathrm{C} \$ 3,231.6$ córdobas (30\% del costo de la canasta básica), debemos mencionar que el sector agropecuario es de uso intensivo en mano de obra, esto hace que sea un sector muy delicado y sensible a las acciones que se tomen en el ajuste de salario mínimo. 
Revista Electrónica de Investigación en Ciencias Económicas

Abriendo Camino al Conocimiento

Facultad de Ciencias Económicas, UNAN-Managua

Los sectores Industria manufacturera, Servicios comunales, Industria Zona Franca y Pesca, están en un intervalo de salario de $C \$ 3,484.2$ el más bajo a $C \$ 3,940.2$ córdobas el más alto. En estos sectores se cubre en promedio un 33\% de la canasta básica. Estos sectores son de uso intenso de mano de obra joven principalmente y se tiene un personal con mayor productividad que el sector agropecuario.

Los sectores Minería, Comercio, Transporte y comunicaciones Electricidad, gas y agua, presenta un salario mínimo similar para $2013, C \$ 4,753.00$ córdobas $(43 \%$ del costo de la canasta básica).Los sectores con un mayor salario mínimo son el sector Construcción y el sector Establecimientos Financieros estos con un monto de C\$ $5,799.00$ (53\% del consto de la canasta básica). El sector Construcción tiene el salario mínimo más alto, debido a la naturaleza de esta actividad económica, el cual es de un gran esfuerzo físico. También el sector Establecimiento Financiero tiene un salario mínimo alto igual al sector construcción, debido a que esté sector demanda personal con un nivel de educación relativamente más alto.

Se observa, que en ninguno de los sectores económicos se cubre el $100 \%$ del Costo de la canasta básica, y lo más lamentable es que todos los sectores no llegan ni a cubrir un $50 \%$ de la canasta básica (a excepción de los trabajadores de los sectores Construcción y Establecimientos Financieros) que tienen el salario mínimo más alto, esto muestra que en Nicaragua, el costo de la canasta básica representa en promedio tres salarios mínimos, un diferencial muy gigantesco, para pequeñas economías como la nicaragüense (Véase anexo 6).

Existe una ley para el salario mínimo. Ley No. 625 "ley de salario mínimo" 2

Arto 4. La ley establece que el salario minio se establecerá cada 6 meses.

Arto 7. La comisión de tripartita y está compuesta por el gobierno, los empleadores y los trabajadores.

\footnotetext{
${ }^{2}$ Gaceta Diario Oficial No. 120 del 26 de junio de 2006.
} 
Revista Electrónica de Investigación en Ciencias Económicas

Abriendo Camino al Conocimiento

Facultad de Ciencias Económicas, UNAN-Managua

Para que la resolución tenga validez deberá ser firmada por lo menos dos de los tres representantes de la comisión.

Arto 8. La comisión Nacional de Salario Mínimo tiene autonomía para fijar el salario mínimo, y establece que debe utilizarse como referencia la inflación acumulada (semestral), el crecimiento según cuentas nacionales, y el objetivo es llegar a cubrir el $100 \%$ de la canasta básica.

Arto11. El periodo de negociación no podrá durar más de dos meses calendarios a partir de su instalación. Transcurrido este término sin que las partes hayan llegado a un acuerdo, el salario mínimo de cada uno de los sectores será fijado por el Ministerio del Trabajo.

Como ya se ha mencionado en párrafos anteriores, al Salario Mínimo en Nicaragua lo determinan las variables Inflación y Crecimiento Económico, la sumatoria de estas variables sirve de parámetro para iniciar las negociaciones entre los agentes económicos (Gobierno, Sindicatos y Sector Privado).

Pero la estimación de inflación y el Crecimiento económico estas sujetas a errores en su medición, por ejemplo la inflación se determina por el IPC (índice de precios al consumidor); este utiliza el índice Laspeyres. El crecimiento económico se calcula a través del PIB real (Producto interno Bruto real); para determinar el PIB real se utiliza el deflactor de PIB, este es un índice que usa la metodología de Paasche.

El índice Laspeyres mide las variaciones del nivel general de los precios comparando el costo de adquisición de una canasta representativa de los bienes y servicios en dos momentos diferentes en el tiempo. La mayor parte de los índices de los precios al consumo (IPC) son índices de Laspeyres.

$$
I L=\frac{\sum_{i=1}^{n} p_{i i} q_{0 i}}{\sum_{i=1}^{n} p_{0 i} q_{0 i}}
$$


Dónde: Pti: precio del bien i en el periodo t, P0i: precio del bien i en el periodo $0 . \mathrm{Y}$ Q0i cantidad del bien i comprado por un hogar típico en el periodo 0. En el índice de Laspeyres, se define la canasta representativa por los q0i, o sea, las cantidades compradas por un hogar típico durante el periodo 0 conocido como el periodo de referencia o año de base.

El deflactor implícito del PIB (DIPIB), por el contrario, utiliza las ponderaciones del año corriente para calcular el índice de precios. Sean las cantidades de los diferentes bienes producidos en el año en curso, definimos el deflactor implícito del PIB así:

Deflactor de PIB $=(\mathrm{PIB}$ Nominal/ PIB Real $){ }^{*} 100$

Este índice de precios es conocido con el nombre de índice de Paasche o ponderado en el año corriente.

$$
I P=\frac{\sum_{i=1}^{n} p_{t i} q_{t i}}{\sum_{i=1}^{n} p_{0 i} q_{t i}}
$$

Tanto el índice de Laspayres usado para el índice de precios al consumidor (IPC) y esta última para calcular la inflación, como el índice de Paasche usado para determinar el Deflactor de PIB; este último para estimar el PIB real que se utiliza para crear el Crecimiento económico, estos índices no tiene mediciones fiables. De hecho, cuando los precios aumentan, el índice de Laspeyres sobrevalora el crecimiento del costo de la vida cuando, al contrario, el índice de Paasche lo subvalora. En el caso inverso, o sea cuando los precios disminuyen, el índice de Laspeyres subvalora la importancia de la disminución del costo de la vida mientras que el índice de Paasche la sobrevalora. 
Esto quiere decir, en palabras más sencillas, que el índice de Laspayres determinara valores de IPC bajos, como la inflación es resultado de una variación en el IPC, el resultado de dicha variación será de un valor de inflación pequeño, ahora bien el índice de Paasche genera un valor muy grande de deflactor del PIB, como el crecimiento económico es resultado de una variación en el PIB real, al ser grande el deflactor del PIB se estimada una valor muy pequeño de PIB real, esto produce un monto de crecimiento económico bajo, esto quiere decir, que el índice de Laspayres genera resultados bajos de inflación y el índice de Paasche determina valores bajos de crecimiento económico. La sumatoria de la inflación más crecimientos económicos da un parámetro bajo para iniciar las negociaciones del salario mínimo.

Con los resultados mencionado en el párrafo anterior los empresarios tienen a su favor un parámetro de negociación bajo, lo que restringe las negociaciones con los sindicatos, este permite a los empresarios a proponer ajustes de salario mínimo con leves variaciones y a los sindicatos a aceptar los ajuste debido que los empresarios tienen a su favor la estimaciones estadísticas de inflación y el crecimiento económico con valores pequeños, es evidente entonces que se producirá un incremento mayor en la brecha de salario mínimo y de costo de la canasta básica, haciendo cada día una utopía un salario mínimo igual al costo de la canasta básica.

Debemos recordar que el Gobierno es el encargado de recopilar y procesar las estadísticas económicas y de elegir las metodologías de cálculo de las variables económicas establecidas por los organismos internacionales como el FMI y el Banco Mundial.

Adicionalmente debemos de tomar en cuenta que existen muchos otros indicadores para medir el IPC, entre ellos tenemos el índice de Fischer que intenta mitigar el problema de la infravaloración o sobrevaloración de los índices de Laspeyres y Paasche, siendo una especie de resultado intermedio de estos. 


$$
I P_{F}=\sqrt{I P_{P} \cdot I P_{L}}
$$

Cabe mencionar que el IPC contiene ciertos criterios considerados muy importantes en donde no se incorpora la introducción de nuevos bienes hasta que se efectúe una actualización en la cesta de productos, esta no añade cambios en la calidad, ni integra una medición del precio de la vivienda en propiedad, así como también pueden variar los resultados si no se realizan debidamente las encuestas y no tiene en cuenta la economía sumergida.

\section{Conclusiones}

Podemos observar que en cada uno de los sectores el costo de la canasta básica representa 3 veces el salario mínimo. Para determinar el Salario mínimo se concluye que el salario mínimo bajo se debe a que la estimación de dichas variables están preparadas estadísticamente para presentar valores bajos; ésta restringe las negociaciones del salario mínimo, concluyendo que el problema no es el alto precio de la Canasta Básica, sino que es el método para estimar el IPC que determina la inflación y también el PIB real que determina el Crecimiento Económico, como posible solución a este inconveniente hacemos referencia a que el índice de Fischer es considerada una alternativa para calcular el IPC y/o el Deflactor del PIB.

\section{Bibliografía}

Monchon, F., Beker, V. (2008). Economía Principios y aplicaciones. (Octava Edición). McGraw-Hill. México DF.

Ministerio del Trabajo (2013): Anuario Estadístico 2012-2013).125 Págs.

Anderson, D. Sweeney, D. (2002). Estadísticas Aplicadas a la Economía y Administración. (10a. Edición).. McGraw-Hill. Madrid España. 
Revista Electrónica de Investigación en Ciencias Económicas

Abriendo Camino al Conocimiento

Facultad de Ciencias Económicas, UNAN-Managua

Monte, O. (2010). Multidimensionalidad de los Números Índices. Revista Análisis Estadístico 10(23):32-36.

Banco Central de Nicaragua (2013): Anuario Estadístico 2012-2013. 85 Págs.

Marx, C. (s.f.). El Capital. En C. Marx, El Capital (pág. 490). Mexico: Tomo I.

REICE | 119

\section{Anexos}

\section{Anexo\#1}

f) Un merpondo de trabalo sin inteventión

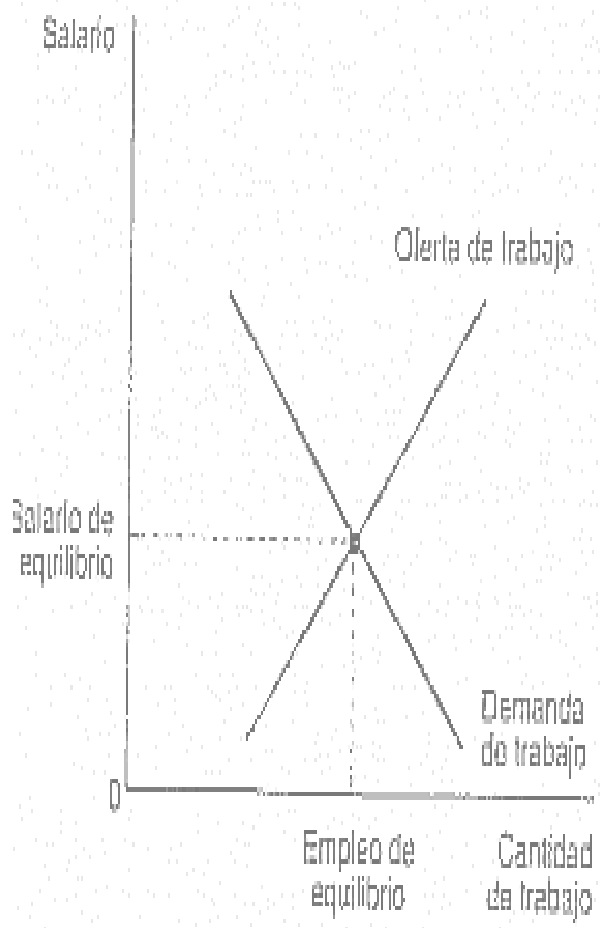

b) Un meterda de frabujo con un 9 alario minhmo ralewante.

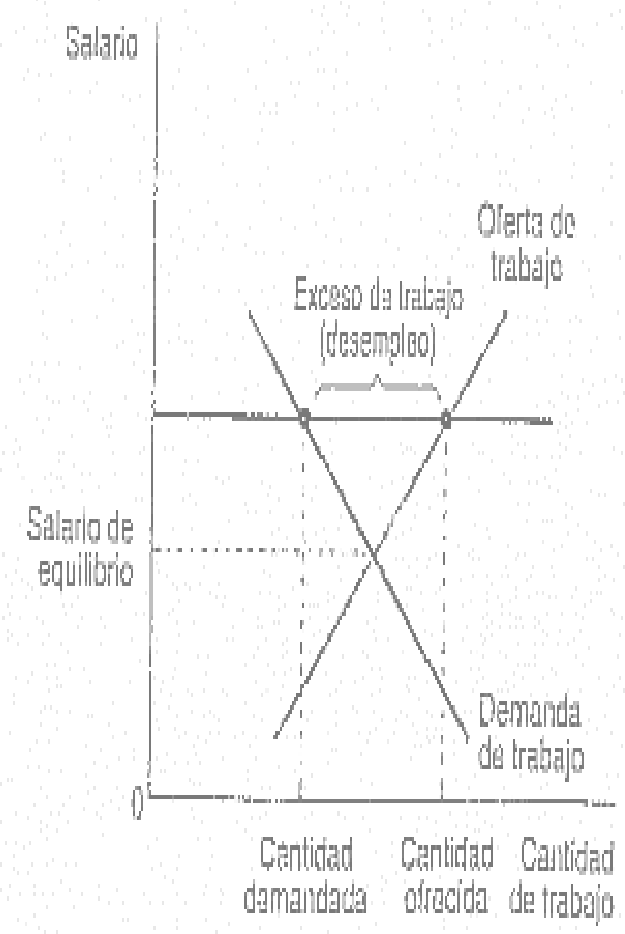

Fuente: Adaptado de Mochón, F., Becker, V. (2008). 
Revista Electrónica de Investigación en Ciencias Económicas

Abriendo Camino al Conocimiento

Facultad de Ciencias Económicas, UNAN-Managua

Anexo\#2

REICE | 120

\section{Proporción de los Componentes de la Canasta Basica del 2012}

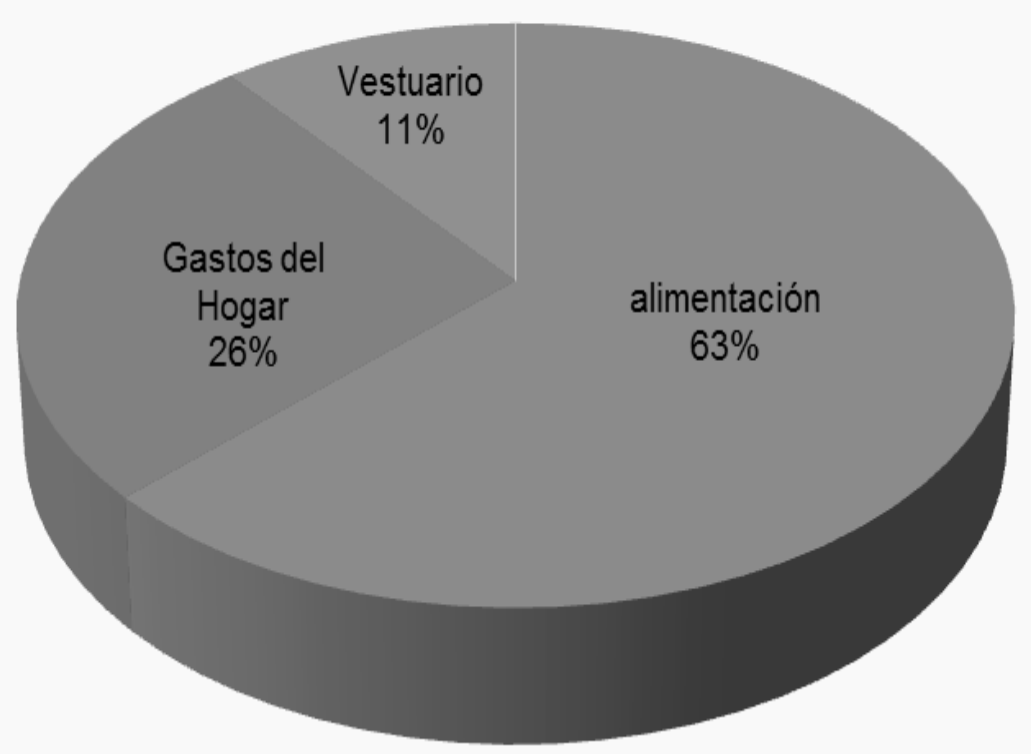

Fuente: Elaboración propia en base a datos de INIDE 
Revista Electrónica de Investigación en Ciencias Económicas

Abriendo Camino al Conocimiento

Facultad de Ciencias Económicas, UNAN-Managua

Anexo \#3

\section{Proporción de los componentes de la Canasta Basica 2013.}

Fuente: Elaboración propia en base a datos de INIDE 
Revista Electrónica de Investigación en Ciencias Económicas

Abriendo Camino al Conocimiento

Facultad de Ciencias Económicas, UNAN-Managua

Anexo\#4

REICE | 122

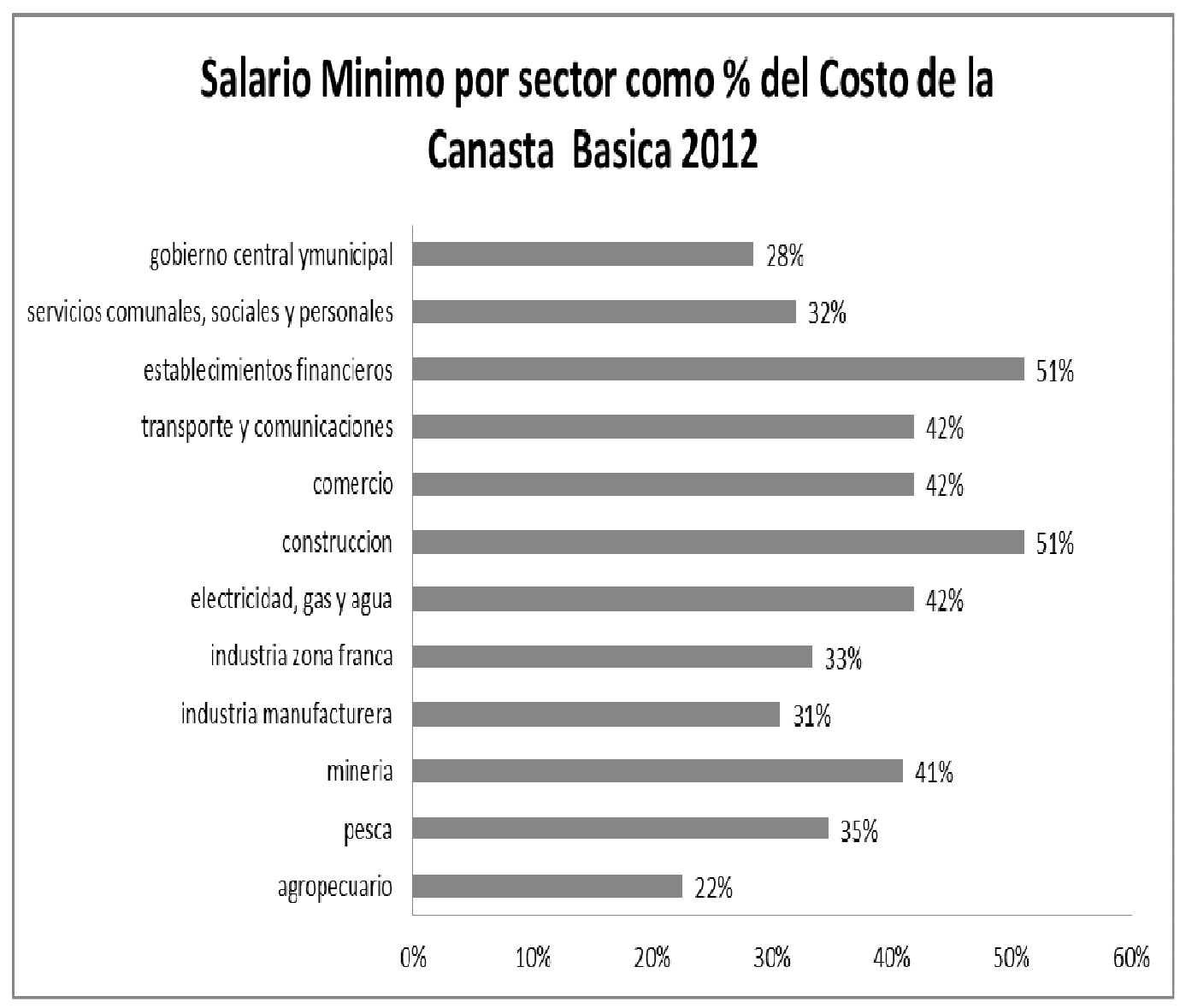

Fuente: Elaboración propia en base a datos del BCN 
Revista Electrónica de Investigación en Ciencias Económicas

Abriendo Camino al Conocimiento

Facultad de Ciencias Económicas, UNAN-Managua

Anexo\#5

REICE | 123

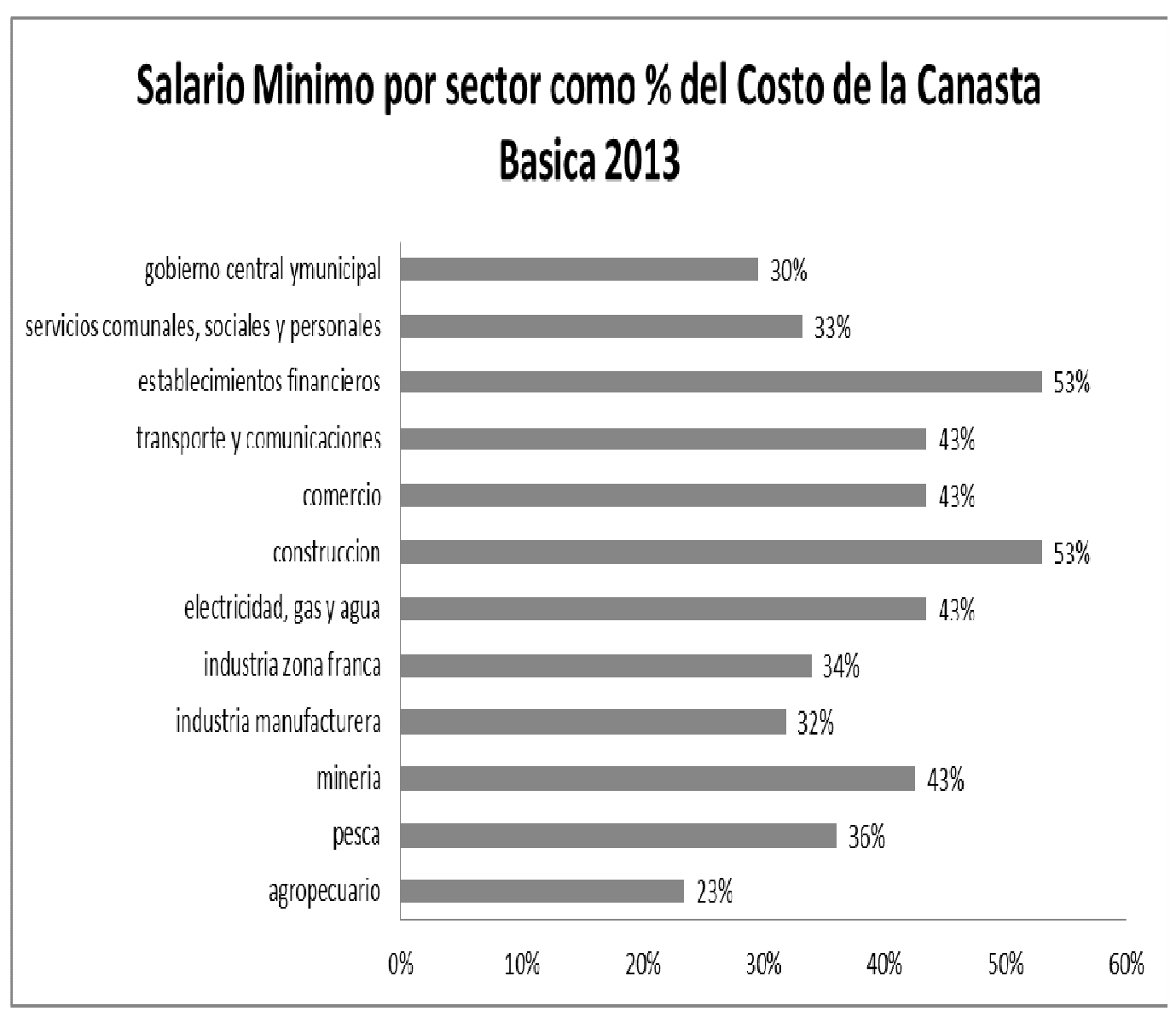

Fuente: Elaboración propia en base a datos del BCN 
Revista Electrónica de Investigación en Ciencias Económicas

Abriendo Camino al Conocimiento

Facultad de Ciencias Económicas, UNAN-Managua

\section{Anexo\#6}

REICE | 124

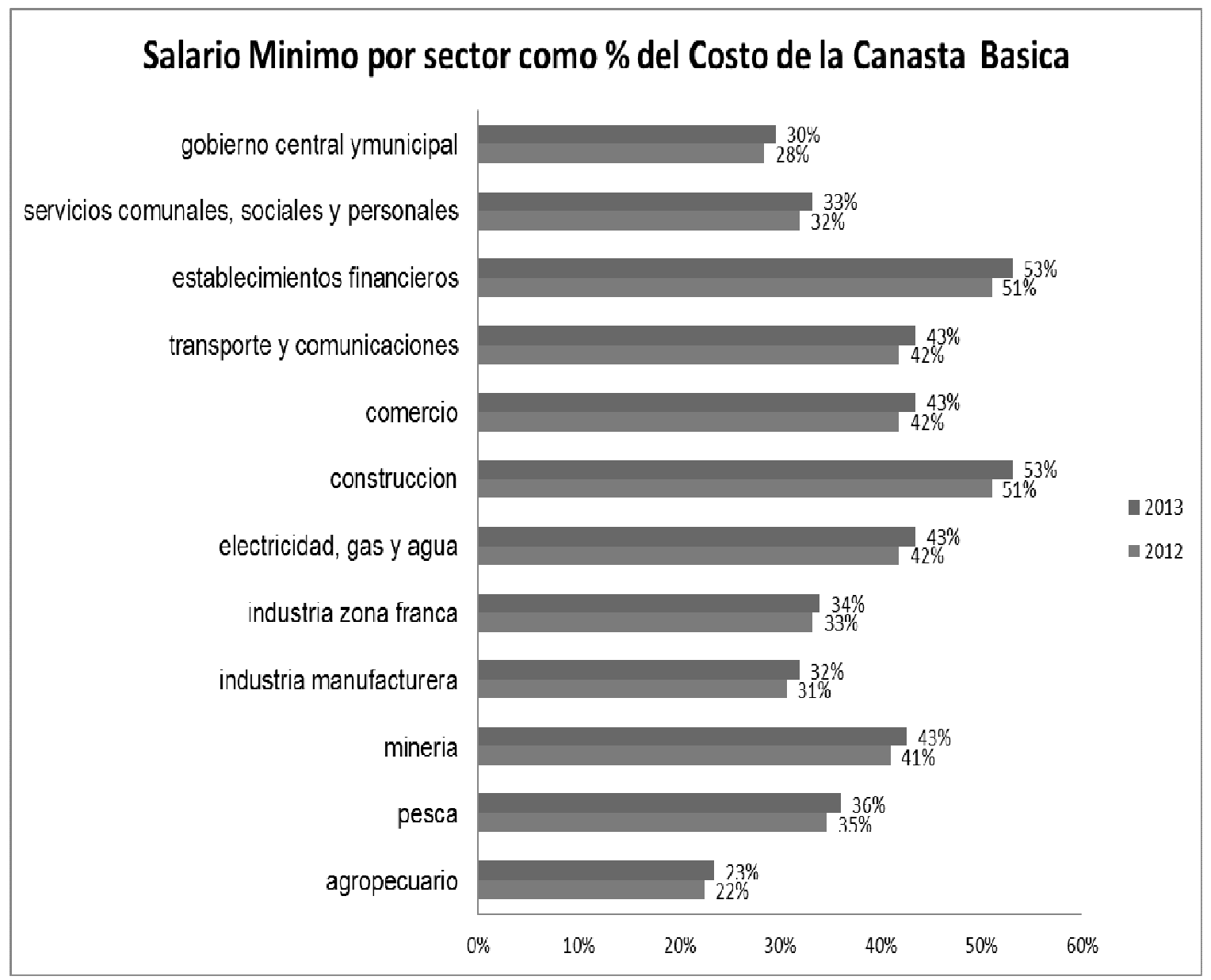

Fuente: Elaboración propia en base a datos del BCN 